\title{
Usando o Paradigma de Equivalência para Aumentar a Correspondência na Fala de Crianças com Implante Coclear na Nomeação de Figuras e na Leitura
}

\author{
Using the Equivalence Paradigm to Increase the Correspondence of Vocalizations \\ by Children with Cochlear Implant in Picture Naming and Reading
}

\author{
Fernanda Luz Anastácio-Pessan ${ }^{a, b}$, Ana Claudia Moreira Almeida-Verdu*, ${ }^{*}$, \\ Maria Cecília Bevilacqua (in memoriam) \& Deisy das Graças de Souza ${ }^{d}$ \\ ${ }^{a}$ Universidade de São Paulo, Bauru, SP, Brasil, ${ }^{b}$ Associação de Pais e Amigos de Excepcionais, Bauru, SP, Brasil, \\ ${ }^{c}$ Universidade Estadual Paulista Júlio de Mesquita Filho, Bauru, SP, Brasil \\ \& ${ }^{d}$ Universidade Federal de São Carlos, São Carlos, SP, Brasil
}

\begin{abstract}
Resumo
Este estudo investigou os efeitos da formação de relações de equivalência entre palavras faladas, palavras impressas e figuras sobre a correspondência na fala de crianças com implante coclear em tarefas de nomeação de figuras e de leitura oral. Antes do estudo as crianças pronunciavam melhor as palavras quando liam do que quando nomeavam as figuras. Foram ensinados dois conjuntos de três relações entre palavras ditadas e as figuras correspondentes, entre as mesmas palavras ditadas e palavras impressas e entre sílabas ditadas e impressas. Sondas periódicas de nomeação das seis figuras e de leitura das palavras impressas, planejadas de acordo com um delineamento de linha de base múltipla entre conjuntos, eram realizadas antes e depois do ensino de cada tipo de relação condicional e do teste de formação de classes de equivalência de cada conjunto. As vocalizações na nomeação de figuras melhoraram progressivamente para cinco dos seis participantes ao longo do ensino do Conjunto 1 e para todos os participantes depois do ensino do Conjunto 2. Na leitura, as vocalizações também ficaram mais precisas. Os resultados estendem descobertas prévias sobre o potencial de procedimentos baseados no paradigma de equivalência de estímulos para a reabilitação da fala e da compreensão auditiva de indivíduos com implante coclear.

Palavras-chave: Nomeação de figuras, leitura, relações de equivalência, deficiência auditiva, implante coclear.
\end{abstract}

\begin{abstract}
The present study investigated the effects of strengthening equivalence relations between spoken words, printed words, and pictures to improve the correspondence between vocalizations in picture naming and in reading tasks in children with cochlear implant. Before the study, the children pronounced words better while reading than while naming pictures. The procedure taught two sets of three relations between dictated words and pictures, between the same dictated words and printed words, and between dictated and printed syllables. Periodic probes evaluated the experimental effects according to a multiple baseline design between stimulus sets. Picture naming and reading of all six words were probed before and after teaching each type of relationship and testing for class formation for each stimulus set. Picture naming progressively improved for five of six participants across teaching and probes with Stimulus Set 1, and for all participants across Stimulus Set 2. Reading scores, initially high, also reached perfect or near perfect accuracy. The results extend previous findings on the potential of procedures based on the stimulus equivalence paradigm for improving speech and listening comprehension of children with cochlear implant.

Keywords: Picture naming, reading, equivalence relations, hearing loss, cochlear implant.
\end{abstract}

* Endereço para correspondência: Avenida Engenheiro Luiz Edmundo Carrijo Coube, 14-01, Vargem Limpa, Bauru, SP, Brasil 17033-360. Fone: (14) 3103-6087. E-mail: fer_anastacio@yahoo.com.br, anaverdu@fc.unesp.br e deisydesouza@gmail.com Os autores são membros do Instituto Nacional de Ciência e Tecnologia, sobre Comportamento, Cognição e Ensino (INCT-ECCE), financiado pelo CNPq (Processo \#573972/2008-7) e pela FAPESP(Processo 2008/57705-8).
A pesquisa foi subsidiada pela FAPESP (\#2009/51798-7) para a segunda autora.

Trabalho conduzido como parte dos requisitos para obtenção do grau de Mestre em Psicologia do Desenvolvimento e Aprendizagem da primeira autora na Universidade Estadual Paulista. As autoras agradecem Carmen Motta Bandini e Raquel Melo Golfeto pela leitura cuidadosa e valiosas sugestões para a conclusão deste manuscrito. 
Repertórios receptivos como reconhecimento e compreensão auditiva são rapidamente adquiridos, em até um ano, após a cirurgia de implante coclear. Porém, a aquisição de repertórios expressivos como a produção e a inteligibilidade da fala não acompanham o mesmo ritmo (Fortunato, Bevilacqua, \& Costa, 2009; Melo, Moret, \& Bevilaccqua, 2008; Mondain et al., 2002; Svirsky, Robbins, Kirk, Pisoni, \& Miyamoto, 2000). Por isso, a aquisição do repertório verbal tem sido considerada crítica para deficientes auditivos usuários de implante coclear.

O paradigma das relações de equivalência oferece uma descrição operacional da linguagem enquanto comportamento simbólico. Estudos em laboratório têm simulado situações cotidianas sob as quais o funcionamento simbólico é adquirido. De maneira geral, crianças com diferentes características de desempenho são ensinadas a estabelecer relações condicionais com um elemento em comum; relacionam condicionalmente figuras (conjunto B) aos seus respectivos nomes ditados (conjunto $\mathrm{A})$, estabelecendo relações $\mathrm{AB}$; e aprendem a relacionar palavras impressas (conjunto $\mathrm{C}$ ) condicionalmente aos mesmos nomes ditados, estabelecendo relações AC. O procedimento de ensino mais frequentemente adotado é o matching-to-sample, pelo qual o participante deve selecionar um estímulo de comparação condicionalmente à apresentação de um estímulo modelo. Como resultado, esse procedimento gera outros comportamentos além daqueles diretamente ensinados, como as relações entre figuras e palavras impressas (BC e CB) que, após o ensino de $\mathrm{AB}$ e $\mathrm{AC}$ evidenciam a formação de classes de equivalência (Sidman, 1971, 1994, 2000; Sidman \& Tailby, 1982). Além de novas relações condicionais emergentes, o procedimento também favorece o desenvolvimento de repertórios verbais de falante (Skinner, 1957) em que respostas vocais (ou gestuais) emitidas pelo aprendiz (representadas por um conjunto D), passam a ocorrer sob controle da palavra impressa (leitura ou relações CD) ou sob controle de figuras ou objetos (tato ou nomeação de figuras - BD).

A aplicação do paradigma de equivalência na investigação da aquisição de significado da estimulação sonora transmitida pelo implante coclear em pessoas com deficiência auditiva é recente. Os primeiros estudos demonstraram que deficientes auditivos cuja privação sonora foi estabelecida antes da fala (pré-linguais) aprendem relações condicionais e formam classes de equivalência com diferentes procedimentos de ensino (fading out e exclusão) e diferentes modalidades de estímulos como pulsos elétricos liberados diretamente na cóclea (da Silva et al., 2006), pseudopalavras (Almeida-Verdu et al., 2008) e palavras convencionais (Almeida-Verdu, Souza, Bevilacqua, \& de Souza, 2009). Estudos mais recentes investigaram condições que favorecem a ampliação dos repertórios verbais dessa população por meio de programas instrucionais baseados no paradigma da equivalência de estímulos. Por exemplo, a inteligibilidade da fala, definida como a correspondência ponto a ponto entre a palavra falada pelo participante e a palavra convencionada pela comunidade verbal, é melhorada quando são fornecidas pistas visuais articulatórias durante o ensino das discriminações (Passarelli et al., 2013) ou quando a topografia vocal é tomada como alvo de ensino direto, como no ensino da resposta ecóica (Almeida-Verdu et al., 2009; Souza, AlmeidaVerdu, \& Bevilacqua, 2013). Verificou-se também que a topografia vocal de participantes leitores tende a ser mais precisa quando é controlada pela palavra escrita do que quando é controlada pela figura (Golfeto, 2010) e que o ensino de relações condicionais auditivo-visuais, apenas, não garante melhora na inteligibilidade da fala (Bataglini, Almeida-Verdu, \& Bevilacqua, 2013).

$\mathrm{O}$ aspecto de interesse neste estudo deriva de um resultado encontrado por Golfeto (2010), que difere daquele tipicamente encontrado nas fases iniciais de aquisição de leitura. Nesse estudo crianças usuárias de implante coclear foram ensinadas a ler por meio de um programa sistemático de ensino de relações entre palavras ditadas e figuras e entre as mesmas palavras ditadas e as palavras impressas correspondentes. Todos os participantes aprenderam as discriminações condicionais ensinadas e demonstraram relações emergentes em testes de formação de classes. Os repertórios de falante (leitura de palavras impressas e nomeação de figuras) foram avaliados por meio de um delineamento de linha de base múltipla entre conjuntos de palavras. Todos os participantes demonstraram aumento na porcentagem de acertos em ambos os repertórios, quando comparados com os dados de pré-teste, porém a inteligibilidade da fala foi maior em tarefas de leitura do que em tarefas de nomeação de figuras. Esse padrão de desempenho foi observado mesmo quando a quantidade de treino das relações condicionais entre palavras ditadas e figuras foi maior do que a quantidade de treino das relações entre palavras ditadas e palavras impressas. Essas diferenças de desempenho podem depender do tipo de controle de estímulos nas tarefas expressivas. Vocalizar na presença de figuras (nomeação) exige um controle pela relação entre a palavra ditada e a figura, enquanto vocalizar na presença da palavra impressa (leitura) envolve outra relação de controle (palavra impressa e palavra ditada); os elementos de uma palavra impressa representam sons e não seu referente e a leitura conta com um controle visual que especifica a topografia vocal que deve ser emitida.

Muitos dos estudos que avaliaram os efeitos do implante coclear sobre a aquisição de repertórios verbais de ouvinte (ou receptivos) e de falante (ou expressivos) identificaram a cirurgia precoce para o implante coclear como uma das principais variáveis relacionadas à aquisição de vocabulário (Geers, Nicholas, \& Moog, 2007; Kirk, Miyamoto, Ying, Perdew, \& Zuganelis, 2002). No entanto, para compreender a melhora no vocabulário é necessário conhecer também as variáveis críticas envolvidas no processo de aquisição da linguagem (Houston, Stewart, Moberly, Hollich, \& Miyamoto, 2012). 
Anastácio-Pessan, F. L., Almeida-Verdu, A. C. M., Bevilacqua, M. C. \& de Souza, D. G. (2015). Usando o Paradigma de Equivalência para Aumentar a Correspondência na Fala de Crianças com Implante Coclear na Nomeação de Figuras e na Leitura.

Embora alguns estudos recentes apontem variáveis relacionadas à aquisição do reconhecimento auditivo de palavras novas (e.g., Almeida-Verdu, Oliveira, Battaglini, Bevilacqua, \& de Souza, 2012; Bataglini et al., 2013; Houston et al., 2012), as relações entre o ouvir e o falar ainda precisam ser esclarecidas. Tomados em conjunto, os estudos sugerem que, na origem, os repertórios de falante e de ouvinte são independentes e que ainda é necessário identificar as condições críticas para promover a interdependência entre o ouvir e o falar, que caracteriza um repertório verbal bem desenvolvido (Greer \& Ross, 2008; Sidman, 1994), tanto em crianças com desenvolvimento típico, de modo geral, quanto em crianças com implante coclear, de maneira particular.

Considerando que, nessa população, o repertório de falante tende a ser mais preciso quando controlado pela palavra impressa do que quando controlado pela figura (e.g., Golfeto, 2010), este estudo teve como objetivo verificar se o controle exercido pela palavra impressa sobre a vocalização de crianças usuárias de implante coclear seria estendido para a figura após o fortalecimento da rede de relações condicionais entre palavra ditada e figura, palavra ditada e palavra impressa e sílaba ditada e sílaba impressa.

\section{Método}

\section{Participantes}

Participaram desse estudo, seis crianças com deficiência auditiva e implante coclear na faixa etária de
11 anos e 4 meses a 14 anos e 2 meses, regularmente matriculadas no Ensino Fundamental. Os pais assinaram um termo de consentimento livre e esclarecido, concordando com a participação do filho ou filha na pesquisa. Todos os procedimentos éticos foram devidamente realizados (Protocolo 106/2009 Serviço de Apoio ao Ensino, Pesquisa e Extensão - Comitê de Ética em Pesquisa [SVAPEPE-CEP]).

A Tabela 1 apresenta as principais características dos participantes, de acordo com os prontuários fornecidos pela instituição na qual recebiam acompanhamento clínico e educacional, após a cirurgia para implante coclear. Dois dos participantes eram meninos e quatro eram meninas, todos com surdez neurossensorial profunda bilateral. O tempo de audição com implante coclear variou de 7 anos a 10 anos e 5 meses até a data do início da pesquisa. Todos os participantes apresentavam desempenho de audição 6 (categoria de audição) e de linguagem 5 (categoria de linguagem), de acordo com dados dos prontuários. A categoria de audição varia de 1 a 6 , e 6 indica que os participantes eram capazes de reconhecer a palavra exclusivamente por meio da audição (Geers, 1994). Quanto à categoria de linguagem, que varia de 1 a 5 , a categoria 5 indica fluência na linguagem oral (Bevilacqua, Delgado, \& Moret, 1996). As atribuições das categorias de audição e linguagem foram feitas por fonoaudiólogos, a partir de avaliações realizadas dois anos e dez meses antes, no máximo, da realização deste estudo.

Tabela 1

Características dos Participantes: Sexo, Idade, Etiologia da Deficiência Auditiva, Tempo de Audição Sem Implante Coclear, Tempo de Audição Com Implante Coclear, Categoria de Linguagem e Categoria de Audição

\begin{tabular}{lccccccc}
\hline Part. & Sexo & Idade & Etiologia & $\begin{array}{c}\text { Tempo de } \\
\text { Audição }\end{array}$ & $\begin{array}{c}\text { Tempo de } \\
\text { Audição (IC) }\end{array}$ & $\begin{array}{c}\text { Categoria } \\
\text { Audição }\end{array}$ & $\begin{array}{c}\text { Categoria } \\
\text { Linguagem }\end{array}$ \\
\hline TAL & F & $11 \mathrm{a} 4 \mathrm{~m}$ & Congênita & 0 & $8 \mathrm{a} 5 \mathrm{~m}$ & 6 & 5 \\
VIV & F & $11 \mathrm{a} 5 \mathrm{~m}$ & Otite (2 meses) & $2 \mathrm{~m}$ & $7 \mathrm{a} 8 \mathrm{~m}$ & 6 & 5 \\
GAB & M & $12 \mathrm{a} 10 \mathrm{~m}$ & Meningite (2a $3 \mathrm{~m})$ & $2 \mathrm{a} 3 \mathrm{~m}$ & $8 \mathrm{a}$ & 6 & 5 \\
GUS & M & $14 \mathrm{a} 2 \mathrm{~m}$ & Congênita & 0 & $10 \mathrm{a} 5 \mathrm{~m}$ & 6 & 5 \\
TAM & F & $11 \mathrm{a} 11 \mathrm{~m}$ & Ototoxidade (9 meses) & $9 \mathrm{~m}$ & $7 \mathrm{a} 11 \mathrm{~m}$ & 6 & 5 \\
LUA & F & $12 \mathrm{a} 1 \mathrm{~m}$ & Neuropatia Auditiva & 0 & $7 \mathrm{a}$ & 6 & 5 \\
\hline
\end{tabular}

\section{Materiais e Estímulos}

O procedimento experimental foi gerenciado por um computador iBookG4, equipado com o software MTS ${ }^{\circledR}$ (Dube, 1991), que também registrava as respostas de seleção realizadas com o mouse. Uma filmadora Sony HadCam registrou as sessões em áudio e vídeo para posterior transcrição e análise do repertorio de fala.
Os estímulos utilizados (figuras e as correspondentes palavras ditadas e palavras impressas) são apresentados na Tabela 2. Foram utilizados dois conjuntos de estímulos em cada modalidade. Os estímulos variaram entre os participantes, com base no critério de ausência ou incorreção na nomeação das figuras, verificados na sonda de vocalização inicial. 
Psicologia: Reflexão e Crítica, 28(2), 365-377.

Tabela 2

Conjunto de Estímulos Utilizados com Cada Participante nas Etapas de Ensino e Teste

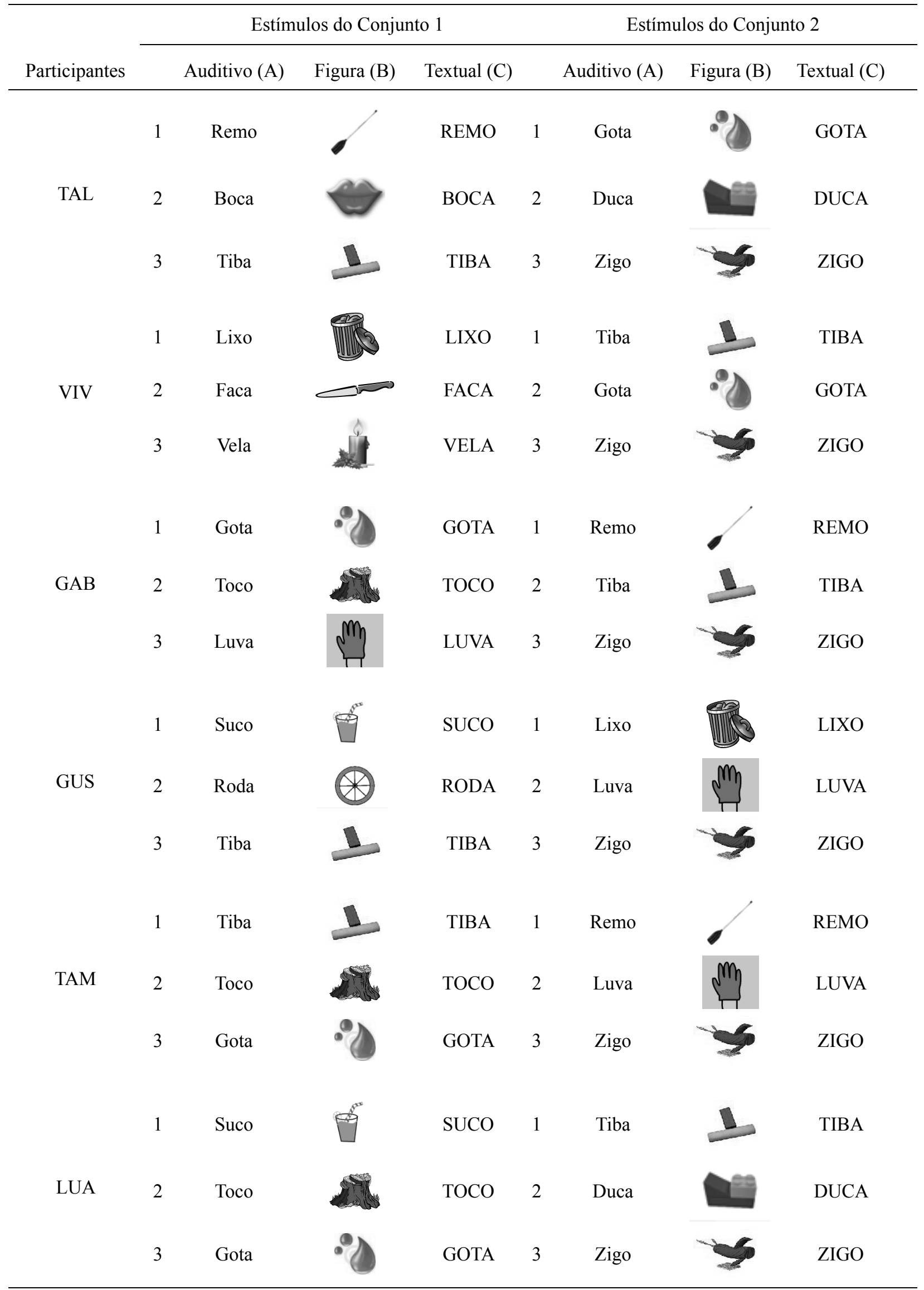


Anastácio-Pessan, F. L., Almeida-Verdu, A. C. M., Bevilacqua, M. C. \& de Souza, D. G. (2015). Usando o Paradigma de Equivalência para Aumentar a Correspondência na Fala de Crianças com Implante Coclear na Nomeação de Figuras e na Leitura.

\section{Procedimento}

O delineamento consistiu no ensino controlado de cada conjunto de estímulos, ou seja, as relações condicionais com estímulos dos Conjuntos 1 e 2 foram ensinadas separadamente e em sequência. $\mathrm{O}$ delineamento de linha de base múltipla entre conjuntos de palavras envolveu medidas de sondas do repertório de falante (nomear as figuras e ler as palavras impressas) que eram conduzidas, sistematicamente, com todos os estímulos dos dois conjuntos. Durante o ensino das relações de seleção com estímulos de um conjunto, as relações de seleção com estímulos do outro conjunto não eram apresentadas, como ilustrado na Figura 1.

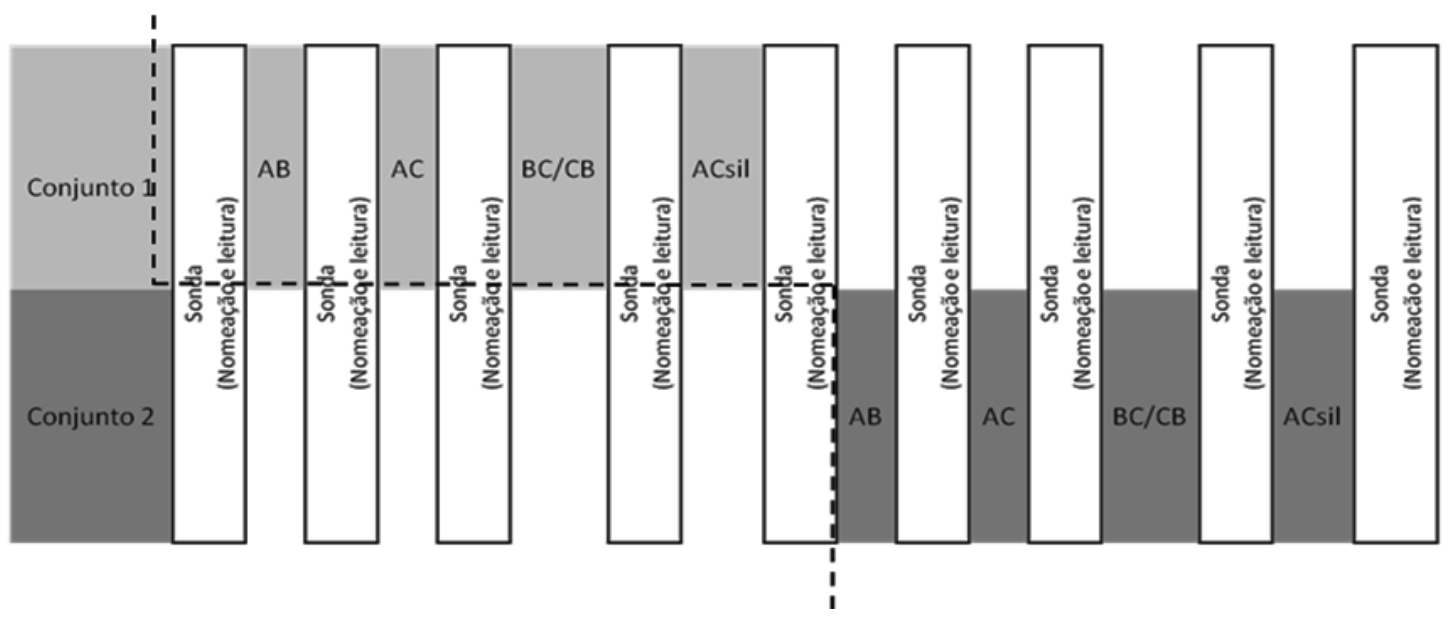

Sondas sucessivas de vocalização

Figura 1. Diagrama ilustrativo do delineamento de linha de base múltipla com sondas de vocalização (barras brancas) antes e depois do ensino de diferentes relações e do teste de formação de classes com estímulos dos Conjuntos 1 (barras cinza claro) e 2 (cinza escuro). Para o Conjunto 1 as sondas depois da fase de ensino são medidas de seguimento; para o Conjunto 2 as sondas antes da fase de ensino são medidas de linha de base.

Nota. Legenda: AB - Emparelhamento entre figuras e palavras ditadas; AC - Emparelhamento entre palavras impressas e palavras ditadas; $\mathrm{BC} / \mathrm{CB}$ - Testes de formação de classes; ACsil - Emparelhamento entre sílabas impressas e sílabas ditadas.

As etapas do procedimento (pré-treino, sondas de vocalizações e ensino de relações auditivo-visuais por emparelhamento com o modelo) estão resumidas na Tabela 3 e melhor descritas a seguir.

Pré-Treino. O objetivo dessa etapa foi ensinar a criança a manusear o mouse do iBook e a realizar a tarefa de matching-to-sample. A tentativa começava com a apresentação de três estímulos de comparação, um em cada vértice do monitor; depois de $2 \mathrm{~s}$ de atraso, o estímulo modelo era apresentado (procedimento de "delayed sample", como descrito por McIlvane, Kledaras, Stoddard, \& Dube, 1990). Inicialmente era conduzido um bloco de nove tentativas de relações condicionais de identidade visual-visual. Posteriormente, um estímulo auditivo era apresentado simultaneamente ao modelo visual, transformando o modelo em um composto. O componente visual do modelo era esmaecido em nove passos de fading out, visando transferir o controle do estímulo modelo de visual para auditivo. O último bloco era constituído de nove tentativas de relações condicionais puramente auditivo-visuais.

\section{Pré-Teste}

Esta etapa teve como objetivo caracterizar o desempenho inicial dos participantes nas relações de seleção, de vocalização e de composição, considerando as diferentes modalidades sensoriais dos estímulos utilizados. Nas tarefas de seleção, cada tentativa apresentava figuras ou palavras impressas na função de estímulos de comparação e palavras ditadas, palavras impressas ou pistas articulatórias na função de estímulo modelo. Nas tarefas de vocalização eram apresentadas figuras ou palavras impressas e o participante era instruído a dizer o nome da figura ou a ler a palavra. Nas tarefas de composição, era solicitada a construção das respostas a partir de sílabas dispostas com função de comparação, após a apresentação de um modelo (palavra ditada, figura ou palavra impressa). Foram testados 30 estímulos, visando selecionar as seis palavras dos dois conjuntos de estímulos para cada participante.

Sondas de Vocalização. Avaliavam a nomeação de figuras e a leitura de palavras impressas selecionadas no Pré-Teste para cada participante (ver Tabela 2). Eram apresentadas 12 tentativas, seis do tipo BD (figura como estímulo discriminativo para a resposta de nomeação) e seis do tipo CD (palavra impressa como estímulo discriminativo para a resposta de leitura oral), em ordem definida por sorteio, sem consequências programadas para acerto ou erro. Essas sondas eram repetidas, sistematicamente, após cada etapa de ensino, como indicado na Tabela 3 e 
Psicologia: Reflexão e Crítica, 28(2), 365-377.

Tabela 3

Etapas de Ensino e Sondas: Relações, Descrição, Número de Tentativas, Destino se Acertos ou Erros

\begin{tabular}{|c|c|c|c|c|c|}
\hline Etapa & Relação & Descrição & $\begin{array}{l}\text { Número } \\
\text { tentativas }\end{array}$ & $\begin{array}{l}\text { Destino } \\
\text { se acertos }\end{array}$ & $\begin{array}{l}\text { Destino } \\
\text { se erros }\end{array}$ \\
\hline \multirow{3}{*}{ Pré-treino } & $\mathrm{BB}$ & Identidade & & & \\
\hline & $\mathrm{AB} / \mathrm{B}$ & Fading out & 18 & Pré-teste & Pré-treino \\
\hline & $\mathrm{AB}$ & Auditivo - visual & & & \\
\hline Pré-teste & & $\begin{array}{c}\text { Seleção } \\
\text { Composição } \\
\text { Vocalização }\end{array}$ & 450 & Ensino & Ensino \\
\hline Sondas & $\mathrm{BD} / \mathrm{CD}$ & Nomeação de figuras e leitura de palavras & 12 & - & - \\
\hline Ensino & $\mathrm{AB}$ & Palavra ditada - figura & 30 & $\begin{array}{c}\text { Sondas de } \\
\text { vocalização }\end{array}$ & $\mathrm{AB}$ \\
\hline Sondas & $\mathrm{BD} / \mathrm{CD}$ & Nomeação de figuras e leitura de palavras & 12 & - & - \\
\hline Ensino & $\mathrm{AC}$ & Palavra ditada-palavra impressa & 30 & $\begin{array}{c}\text { Sondas de } \\
\text { vocalização }\end{array}$ & $\mathrm{AC}$ \\
\hline Sondas & $\mathrm{BD} / \mathrm{CD}$ & Nomeação de figuras e leitura de palavras & 12 & - & - \\
\hline Revisão & $\mathrm{AB} / \mathrm{AC}$ & $\begin{array}{l}\text { Palavra ditada - figura/ Palavra ditada - } \\
\text { palavra impressa }\end{array}$ & 12 & $\begin{array}{c}\text { Sondas de } \\
\text { vocalização }\end{array}$ & Revisão \\
\hline \multirow{2}{*}{$\begin{array}{l}\text { Teste de } \\
\text { equivalência }\end{array}$} & $\mathrm{BC}$ & Figura - palavra impressa & 18 & \multirow{2}{*}{$\begin{array}{c}\text { Sondas de } \\
\text { vocalização }\end{array}$} & \multirow{2}{*}{$\begin{array}{c}\text { Sondas de } \\
\text { vocalização }\end{array}$} \\
\hline & $\mathrm{CB}$ & Palavra impressa - figura & 18 & & \\
\hline Sondas & $\mathrm{BD} / \mathrm{CD}$ & Nomeação de figuras e leitura de palavras & 12 & - & - \\
\hline Ensino & ACsil & Sílaba ditada - sílaba impressa & 36 & $\begin{array}{c}\text { Sondas de } \\
\text { vocalização }\end{array}$ & ACsil \\
\hline Sondas & $\mathrm{BD} / \mathrm{CD}$ & Nomeação de figuras e leitura de palavras & 12 & - & - \\
\hline
\end{tabular}

Nota. A mesma sequência de procedimentos era realizada com os estímulos do Conjunto 1 e do Conjunto 2.

ilustrado na Figura 1; a cada repetição um novo sorteio determinava a sequência das tentativas.

Ensino de Seleção de Figuras, de Palavra Impressa e de Sílaba Impressa sob Controle Auditivo. Os participantes foram ensinados a selecionar figuras, palavras impressas e sílabas sob controle dos estímulos auditivos correspondentes, em um procedimento de emparelhamento com o modelo simultâneo. Cada tipo de relação era ensinado separadamente (ver Tabela 3 e Figura 1). A sequência experimental foi aplicada com os estímulos do Conjunto 1 e repetida com os estímulos do Conjunto 2 .

Uma tentativa de ensino era iniciada com a apresentação de modelo auditivo pelo auto-falante do computador simultaneamente à apresentação de um quadrado azul no centro da tela. Após um clique com o mouse no quadrado azul, que funcionava como uma resposta de observação ao estímulo auditivo, estímulos visuais (figuras, palavras impressas ou sílabas) eram apresentados como comparações em três dos quatro vértices da tela do computador. O vértice vazio mudava de posição ao longo das tentativas. Os três estímulos de comparação ficavam expostos até que o participante selecionasse um estímulo de comparação ou por até $16 \mathrm{~s}$ (na ausência de resposta). Consequências diferenciais eram programadas para acerto e erro. Respostas corretas eram seguidas por estrelas coloridas na tela e um jingle; e respostas incorretas eram seguidas por uma tela preta por $2 \mathrm{~s}$, seguida de uma tela de intervalo entre tentativas (IET) por $2 \mathrm{~s}$. Caso o participante não emitisse uma resposta de seleção, a tentativa era computada como erro, a tela de intervalo entre tentativas era apresentada e uma nova tentativa era iniciada. Ao longo de sucessivas tentativas, os estímulos de comparação eram aleatorizados nas posições que ocupavam nos vértices da tela do computador. 
Anastácio-Pessan, F. L., Almeida-Verdu, A. C. M., Bevilacqua, M. C. \& de Souza, D. G. (2015). Usando o Paradigma de Equivalência para Aumentar a Correspondência na Fala de Crianças com Implante Coclear na Nomeação de Figuras e na Leitura.

Relações $A B$. O ensino iniciava com a exposição do participante a um bloco da relação entre palavra ditada e figura $(\mathrm{AB})$, composto por nove tentativas com três relações de linha de base definidas no Pré-teste (X1Y1, $\mathrm{X} 2 \mathrm{Y} 2, \mathrm{X} 3 \mathrm{Y} 3$ ). Após a exposição a esse bloco, caso o participante obtivesse $100 \%$ de acertos, era exposto ao ensino de três relações condicionais auditivo-visuais novas (A1B1, A2B2, A3B3) com estímulos do Conjunto 1. Para o ensino destas relações foi empregado o procedimento de emparelhamento por exclusão (Dixon, 1977; McIlvane \& Stoddard, 1981).

No ensino por exclusão, diante de um estímulo modelo novo (A1) eram apresentados, como estímulos de comparação, dois estímulos de linha de base (Y1, Y2 ou Y3) convencionados experimentalmente como S- e um estímulo novo (B1), convencionado experimentalmente como estímulo discriminativo ( $\mathrm{S}+$ ); as tentativas de ensino eram seguidas por três tentativas controle da mesma relação, isto é, o estímulo de comparação novo (B1) era apresentado como uma alternativa de escolha, juntamente com dois comparações da linha de base, mas o estímulo modelo era um estímulo da linha de base; se o desempenho estivesse sob controle das relações ensinadas na linha de base, a resposta correta seria a seleção do estímulo correspondente e não de $\mathrm{B} 1$; a mesma rotina experimental foi adotada para o ensino das relações A2B2 e A3B3.

Por fim, eram apresentadas nove tentativas das três relações entre palavra ditada e figura (A1B1, A2B2 e A3B3) nas quais eram apresentados, como estímulos de comparação, os três estímulos novos (B1, B2 e B3). Esse tipo de tentativas é denominado de tentativas de aprendizagem; respostas corretas indicam que o participante aprendeu a relação entre cada estímulo modelo e o estímulo de comparação definido como correto (A1B1, A2B2, A3B3), sem o apoio de estímulos de linha de base que permitam o responder por exclusão. Um bloco de tentativas de ensino das três relações condicionais totalizava 30 tentativas distribuídas entre tentativas de linha de base, de exclusão, de controle e de aprendizagem.

Relações AC. O mesmo procedimento descrito para o ensino das relações $\mathrm{AB}$ foi adotado para o ensino das relações entre palavra ditada e palavra impressa (AC).

Relações ACsil. O mesmo procedimento descrito para o ensino das relações $\mathrm{AB}$ também foi adotado para o ensino das relações entre sílaba ditada e sílaba impressa (ACsil).

Teste de Formação de Classes (BC e CB). Pretendeu verificar se as funções discriminativas exercidas pelas palavras faladas se estenderiam para as figuras e palavras impressas e se essas seriam relacionáveis entre si. Antes de realizar o teste, foi realizado um bloco de tentativas que misturavam as relações $\mathrm{AB}$ e $\mathrm{AC}$; esse bloco era repetido, até que o participante apresentasse $100 \%$ de acertos, como linha de base estável para os testes de equivalência. Estes testes avaliaram se, após haver selecionado duas figuras diferentes (por exemplo, B1 e C1) quando uma mesma palavra era ditada (por exemplo, A1), o participante relacionaria essas duas figuras (B1C1 e C1B1), sem exposição prévia a esta tarefa. Esta etapa avaliou, então, a extensão do controle dos estímulos sonoros para os estímulos visuais pelo teste das relações condicionais visuais-visuais $(\mathrm{B} 1 \mathrm{C} 1$, $\mathrm{B} 2 \mathrm{C} 2, \mathrm{~B} 3 \mathrm{C} 3$ e C1B1, C2B2, C3B3). O bloco de teste era composto por 24 tentativas: nove tentativas de relações BC e nove tentativas de relações $\mathrm{CB}$, mescladas com tentativas de linha de base: três relações $\mathrm{AB}$ (A1B1, A2B2, A3B3) e três $\mathrm{AC}(\mathrm{A} 1 \mathrm{C} 1, \mathrm{~A} 2 \mathrm{C} 2, \mathrm{~A} 3 \mathrm{C} 3)$. Os diferentes tipos de tentativas eram misturados e o bloco tinha programação para ser aplicado apenas uma vez, independente do número de acertos. Durante o teste, respostas nas tentativas de linha de base (relações $\mathrm{AB}$ e AC) eram consequenciadas, mas respostas nas tentativas de teste não tinham consequências programadas.

As sondas de vocalização foram repetidas sistematicamente ao longo de todas as etapas de ensino, como ilustrado na Figura 1. Sondas realizadas antes do ensino com os estímulos de um conjunto eram consideradas sondas de linha de base, enquanto as realizadas depois do ensino eram consideradas sondas de retenção. Só foram realizadas sondas de retenção com estímulos do Conjunto 1.

\section{Análise dos Dados de Vocalização}

A palavra inteira pode ser uma unidade de análise muito extensa para evidenciar progressos na fala dos participantes, especialmente no caso desta população. Mesmo que a palavra inteira seja considerada incorreta, a inspeção dos registros pode revelar graus variados de aproximação em relação à fala padrão. Uma análise que permite capturar progressos em unidades menores que a palavra foi sugerida por Lee e Sanderson (1987; cf. Hanna, de Souza, de Rose, \& Fonseca, 2004) para análise da escrita. Esta medida, que foi adaptada por Almeida-Verdu et al. (2012), para análise da fala, consiste em um escore baseado na porcentagem de bigramas corretos, em cada palavra falada (sequências de dois fonemas consecutivos na fala, correspondentes aos grafemas da palavra escrita na transcrição). Por exemplo, a palavra escrita BOLA tem cinco bigramas (-b / bo / o l/ la / a-); o traço indica espaços sem som antes e depois da palavra. Quando se computa 0 ou 1 para respostas corretas e incorretas, respectivamente, se a criança fala "ola", em vez de bola, o escore é zero; mas pela análise dos bigramas, ela teria acertado os três bigramas finais, obtendo um escore de $60 \%$, que indica uma aproximação da fala correta. Esta análise foi aplicada ao conjunto dos dados de fala do presente estudo, para a análise guiada pelo delineamento de linha de base múltipla.

Os dados de vocalização foram transcritos e foi calculada a porcentagem de bigramas emitidos corretamente nas sucessivas sondas de nomeação de figuras e de leitura de palavras impressas. As transcrições foram realizadas por uma das pesquisadoras (ACAV) e por um observador independente para $50 \%$ das tentativas de todos os participantes. A concordância entre observadores foi calculada de acordo com a fórmula (A/A+D) x 100 (Kazdin, 1982), onde $\mathrm{A}$ indica acordos e $\mathrm{D}$ indica desacordos. A porcentagem de acordos foi $80,1 \%$. 


\section{Resultados}

Os resultados foram organizados pelo número de exposições aos blocos de tentativas até a obtenção do critério de acertos estabelecido para as tarefas de seleção de estímulos e pela porcentagem de acertos de bigramas em cada uma das sondas de vocalização nas tarefas de nomeação de figuras e de leitura das palavras impressas dos Conjuntos 1 e 2. Porcentagens de acertos nas fases de ensino e de testes de formação de classes não são apresentadas porque todos os participantes aprenderam as discriminações condicionais e formaram classes entre as figuras e as respectivas palavras ditadas e impressas.
Relações Condicionais Baseadas em Seleção

A Tabela 4 apresenta o número de exposições aos blocos de pré-treino, seleção de figuras (AB), palavras (AC) e sílabas (ACsil) impressas sob controle da palavra ou da sílaba ditada até o critério estabelecido e, também, aos testes de formação de classes (BC/CB), para os quais não havia critério. Como pode ser observado, todos os participantes aprenderam as relações do Pré-treino necessitando de apenas uma exposição aos blocos de tentativas programados; ainda que quatro participantes (Tam, Viv, Gus e Tal) tenham apresentado erros nos blocos de relação condicional visual-visual e de fading out do componente visual do modelo, todos os participantes apresentaram $100 \%$ de acertos nos blocos com tentativas puramente auditivo-visuais, necessitando de apenas uma exposição a esse bloco de tentativas.

Tabela 4

Número de Exposições de Cada Participante aos Blocos de Pré-Treino, Ensino de Discriminações Condicionais e Testes de Equivalência até a Obtenção do Critério de Acertos

\begin{tabular}{|c|c|c|c|c|c|c|c|c|c|}
\hline \multirow[b]{2}{*}{ Participante } & \multicolumn{3}{|c|}{ Pré-treino } & & \multirow[b]{2}{*}{$\mathrm{AB}$} & \multirow[b]{2}{*}{$\mathrm{AC}$} & \multirow[b]{2}{*}{$\mathrm{AB} / \mathrm{AC}$} & \multirow[b]{2}{*}{$\mathrm{BC} / \mathrm{CB}$} & \multirow[b]{2}{*}{ ACsil } \\
\hline & $\begin{array}{l}\text { Visual } \\
\text { Visual }\end{array}$ & Fading Out & $\begin{array}{c}\text { Auditivo } \\
\text { Visual }\end{array}$ & & & & & & \\
\hline \multirow[t]{2}{*}{ TAL } & $1^{\mathrm{a}}$ & $1^{\mathrm{a}}$ & 1 & Conj. 1 & 4 & 1 & 1 & 1 & $1^{\mathrm{a}}$ \\
\hline & & & & Conj. 2 & 1 & 1 & 1 & 1 & $1^{\mathrm{a}}$ \\
\hline \multirow[t]{2}{*}{ VIV } & $1^{\mathrm{a}}$ & 1 & 1 & Conj. 1 & 1 & 1 & 1 & $2^{\mathrm{c}}$ & $1^{\mathrm{a}}$ \\
\hline & & & & Conj. 2 & 1 & 1 & 1 & 1 & 1 \\
\hline \multirow[t]{2}{*}{ GAB } & 1 & 1 & 1 & Conj. 1 & 1 & 1 & 1 & 1 & $1^{\mathrm{a}}$ \\
\hline & & & & Conj. 2 & 1 & 1 & 1 & 1 & 1 \\
\hline \multirow[t]{2}{*}{ GUS } & $1^{a}$ & $1^{\mathrm{a}}$ & 1 & Conj. 1 & 1 & 1 & 1 & 1 & $1^{\mathrm{a}}$ \\
\hline & & & & Conj. 2 & 1 & 1 & 1 & 1 & 1 \\
\hline \multirow[t]{2}{*}{ TAM } & $1^{\mathrm{a}}$ & 1 & 1 & Conj. 1 & $1^{\mathrm{a}}$ & $1^{\mathrm{a}}$ & 1 & $-\mathrm{b}$ & $1^{\mathrm{a}}$ \\
\hline & & & & Conj. 2 & 1 & 1 & 2 & 1 & $1^{\mathrm{a}}$ \\
\hline \multirow[t]{2}{*}{ LUA } & 1 & 1 & 1 & Conj. 1 & 1 & 1 & 1 & $1^{\mathrm{a}}$ & $1^{\mathrm{a}}$ \\
\hline & & & & Conj. 2 & 2 & 1 & 1 & 1 & $1^{\text {a }}$ \\
\hline
\end{tabular}

${ }^{a}$ Blocos com no máximo1 erro não eram repetidos.

${ }^{\mathrm{b}}$ Por um erro de programação, a participante TAM não realizou este teste.

${ }^{\mathrm{c}}$ A participante VIV obteve $100 \%$ de acertos somente na segunda exposição aos testes BC/CB.

Em relação ao fortalecimento de relações auditivovisuais $(\mathrm{AB}, \mathrm{AC}, \mathrm{ACsil})$ todos os participantes necessitaram de apenas uma exposição aos blocos de tentativas até demonstrarem $100 \%$ de acertos com pelo menos um conjunto de palavras. Somente dois participantes necessitaram de mais de uma exposição aos blocos programados para demonstrar precisão na relação entre palavra ditada e figura; LUA necessitou de duas exposições ao bloco de relações auditivo-visuais com palavras do Conjunto 2 (que incluía três pseudopalavras e as correspondentes figuras abstratas) e TAL necessitou de quatro exposições com palavras do Conjunto 1 . Todos os participantes formaram 
Anastácio-Pessan, F. L., Almeida-Verdu, A. C. M., Bevilacqua, M. C. \& de Souza, D. G. (2015). Usando o Paradigma de Equivalência para Aumentar a Correspondência na Fala de Crianças com Implante Coclear na Nomeação de Figuras e na Leitura.

classes logo na primeira exposição aos testes de relações de equivalência, exceto as participantes TAM que, por um erro de programação, não foi exposta aos referidos testes e VIV que foi exposta duas vezes ao mesmo bloco até que obtivesse $100 \%$ de acertos nas relações BC/CB.

\section{Sondas de Vocalizações}

A porcentagem de acertos dos participantes nas sucessivas sondas de vocalização é apresentada na Figura 2. A porcentagem de acertos foi calculada de acordo com bigramas após a transcrição das vocalizações; $100 \%$ de acertos significa que o participante acertou todos os bigramas e, consequentemente, todas as palavras inteiras; valores menores que $100 \%$ indicam aproximações à resposta correta e podem significar acertos intermediários em todas as palavras ou uma combinação entre palavras pronunciadas corretamente e palavras pronunciadas com algum grau de acerto.

De acordo com a Figura 2, à exceção de GUS, todos os outros cinco participantes apresentaram porcentagens mais baixas em nomeação de figuras do que em leitura na avaliação de pré-teste. GUS apresentou porcentagens elevadas de acertos nas duas tarefas, mas os acertos em nomeação foram ligeiramente maiores que os de leitura para as palavras do Conjunto 1 . Após a exposição às etapas de ensino, todos os participantes apresentaram melhora substancial das vocalizações sob controle dos estímulos de um ou dos dois conjuntos, quando se compara a porcentagem de acertos com a do pré-teste. O melhor desempenho em leitura pode se dever, pelo menos em parte, ao uso de pseudopalavras (do total de seis palavras, duas ou três eram pseudopalavras - ver Tabela 2) e de figuras abstratas sem nome em português. As palavras impressas eram dissílabas e os participantes poderiam decodificá-las; ao contrário, seria difícil atribuir o nome correto (arbitrariamente definido no experimento) a uma figura abstrata.

As medidas tomadas de acordo com o delineamento de linha de base múltipla permitem examinar o curso da evolução das vocalizações após o ensino ou fortalecimento das relações com os estímulos de cada conjunto. Alguns participantes apresentaram porcentagens de acertos elevadas no pré-teste de pelo menos um dos conjuntos de estímulos (geralmente para o Conjunto 1, que incluía palavras/figuras familiares), mas ainda assim a descrição das mudanças nos desempenhos individuais sugere aspectos importantes para discussão.

Como mostra o primeiro painel na coluna esquerda da Figura 2, no pré-teste TAL lia com elevada precisão as palavras dos dois conjuntos; porém a porcentagem de nomeação de figuras foi intermediária para o Conjunto 1 e bastante baixa para o Conjunto 2 (duas das três figuras eram abstratas). Após o ensino da relação $\mathrm{AB}$ (palavra ditada-figura) do Conjunto 1, ocorreu melhora nas vocalizações nas duas tarefas para os estímulos desse conjunto e as porcentagens permaneceram elevadas ao longo de todas as etapas seguintes. Para os estímulos do Conjunto 2 , que estavam sendo mantidos em linha de base, a lei- tura foi mantida acurada e ocorreu aumento gradual na nomeação de figuras, embora tais estímulos não fossem incluídos no ensino. Quando foram ensinadas as relações com o Conjunto 2, os ganhos obtidos na fase anterior foram mantidos e o participante passou a nomear as figuras com $100 \%$ de acertos, igualando os escores na fala para as duas tarefas. Padrões similares de mudanças foram observados também nos dados de VIV (painel superior na coluna da direita). Para essa participante também foram usadas duas pseudopalavras no Conjunto 2 .

$\mathrm{O}$ painel no centro da coluna esquerda mostra que no pré-teste $\mathrm{GAB}$ acertou entre 80 e $90 \%$ na tarefa de leitura, mas acertou muito pouco na nomeação de figuras dos dois conjuntos $(10 \%$ de acertos tanto para palavras do Conjunto 1 quanto do Conjunto 1). Após o ensino das relações palavra ditada-figura (AB) para o Conjunto 1 , as vocalizações se aproximaram de $100 \%$ de acertos tanto para leitura quanto para a nomeação das figuras desse conjunto e os acertos permaneceram nesse patamar ao longo das fases seguintes. Como deveria ser esperado, caso os repertórios em relação aos dois conjuntos de estímulos fossem independentes, a nomeação das figuras do Conjunto 2 (que incluía duas pseudopalavras, TIBA e ZIGO) continuou baixa após o ensino tanto de $\mathrm{AB}$ quanto de $\mathrm{AC}$ com os estímulos do Conjunto 1. Contudo, após incluir os estímulos do Conjunto 1 em classes de equivalência, a nomeação das figuras do Conjunto 2 melhorou substancialmente, atingindo porcentagens de acertos próximas de $90 \%$. Quando a relações do Conjunto 2 foram diretamente ensinadas, a nomeação de figuras tendeu a se tornar ainda mais precisa e permaneceu assim nas sondas subsequentes.

O participante GUS (painel na linha central da coluna à direita) apresentava escores elevados, embora abaixo de $100 \%$, para ambos os operantes verbais. O ensino das relações do Conjunto 1 resultou em aumento nos acertos em leitura (que era mais baixo para este participante, com os estímulos desse conjunto), mas não melhorou a nomeação de figuras; pelo contrário, após o ensino das relações condicionais com sílabas, a nomeação piorou. Para os estímulos do Conjunto 2, a leitura foi mantida e a nomeação melhorou como função do ensino com os estímulos do Conjunto 1, mas também diminuiu após o ensino da relação envolvendo sílabas. Quando o Conjunto 2 passou a ser ensinado, a leitura das palavras dos dois conjuntos foi mantida próxima de $100 \%$, enquanto a nomeação diminuiu para as figuras dos dois conjuntos. Após o ensino de $\mathrm{AC}$ e do teste de equivalência, os acertos em nomeação aumentaram para os dois conjuntos e o aluno concluiu o procedimento com aproximadamente $100 \%$ de acertos em todas as vocalizações.

A participante TAM (painel inferior na coluna esquerda) apresentou $100 \%$ de acertos em leitura para as palavras dos dois conjuntos no pré-teste e manteve essa porcentagem, ao longo de todo o procedimento, para as palavras do Conjunto 1. As nomeações eram baixas (ao redor de $40 \%$ ) para as figuras dos dois conjuntos (para essa participante foi empregada apenas uma pseudopalavra 
Retenção
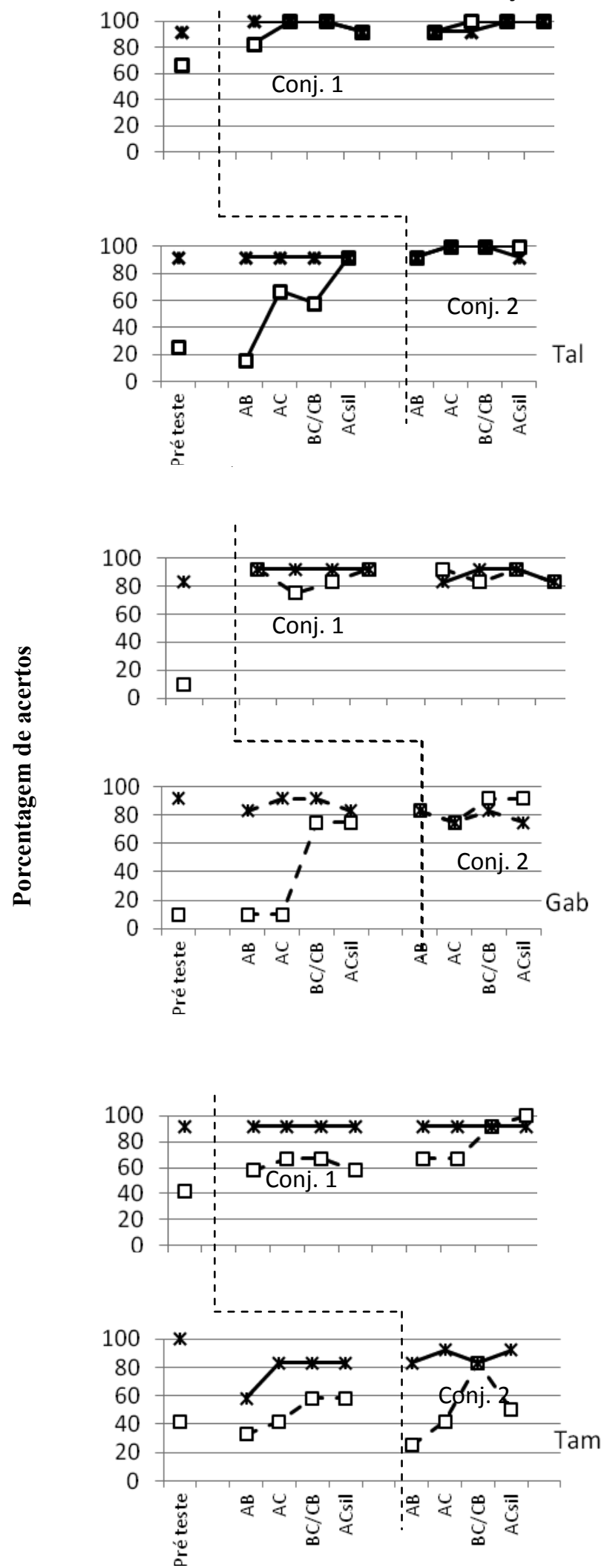
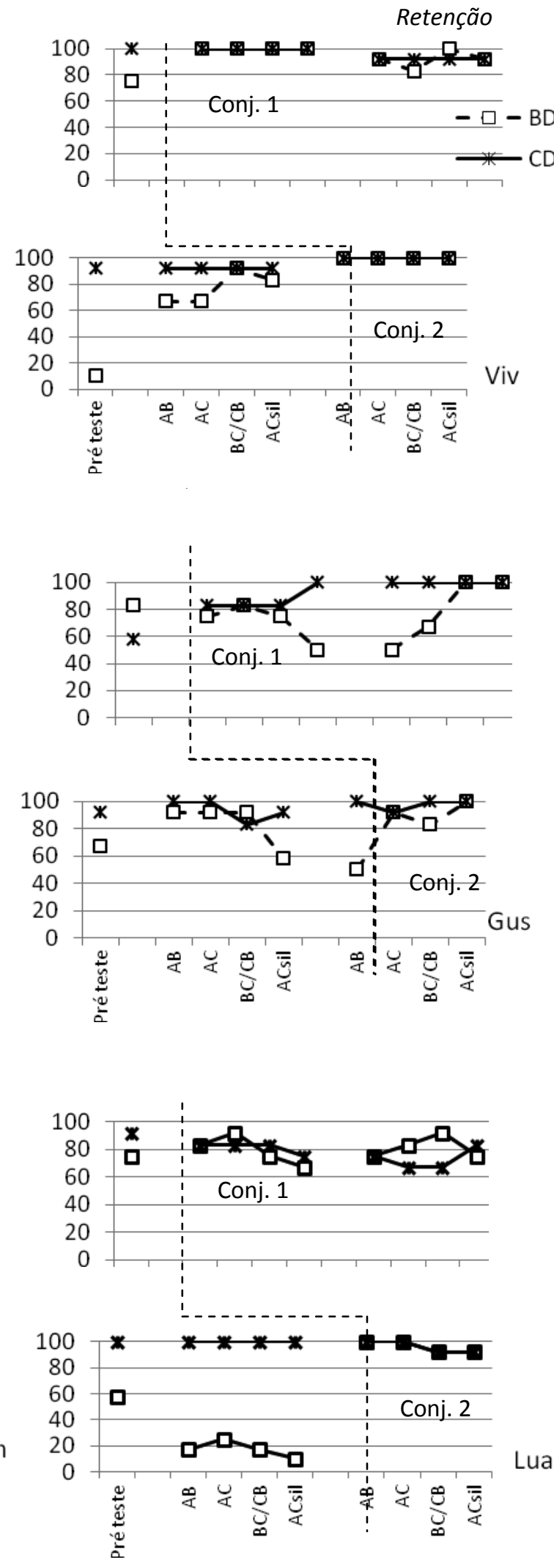

\section{Sondas sucessivas de vocalização}

Figura 2. Porcentagem de acertos de participantes individuais nas sucessivas sondas de nomeação de figuras (BD - quadrados vazios) e de leitura oral ( $C D$ - asteriscos) das três palavras de cada um dos dois conjuntos. As sondas eram realizadas após a etapa de ensino de cada uma das relações indicadas no eixo de X (Ver Figura 1). Para cada participante o gráfico do painel superior representa os dados com estímulos do Conjunto 1 e o painel inferior os dados com estímulos do Conjunto 2. Pontos à esquerda da linha tracejada correspondem a sondas antes do ensino com os estímulos do respectivo conjunto. 
Anastácio-Pessan, F. L., Almeida-Verdu, A. C. M., Bevilacqua, M. C. \& de Souza, D. G. (2015). Usando o Paradigma de Equivalência para Aumentar a Correspondência na Fala de Crianças com Implante Coclear na Nomeação de Figuras e na Leitura.

em cada conjunto, uma vez que ela não nomeava figuras familiares no pré-teste). Após o ensino de $\mathrm{AB}$ entre estímulos do Conjunto 1, a nomeação das figuras aumentou para aproximadamente $60 \%$ para este conjunto, mas a leitura das palavras do Conjunto 2 diminuiu para porcentagens próximas às encontradas para a nomeação. A nomeação das figuras de ambos os conjuntos permaneceu próxima $60 \%$ para os dois conjuntos ao longo das etapas de ensino para o Conjunto 1, enquanto a leitura das palavras do Conjunto 2 aumentou para $80 \%$ após o ensino de $\mathrm{AC}$ e permaneceu nesse patamar. Quando foi iniciada a fase de ensino com os estímulos do Conjunto 2, a nomeação das figuras dos dois conjuntos melhorou progressivamente e esta participante também concluiu o procedimento com escores próximos de $100 \%$ de acertos, exceto na última fase, em que a nomeação das palavras do Conjunto 2 diminuiu.

$\mathrm{O}$ último painel à direita mostra que a participante LUA manteve escores entre 60 e $100 \%$ tanto para a leitura quanto para a nomeação de estímulos do Conjunto 1, terminando o procedimento com $80 \%$ de acertos. A nomeação das figuras do Conjunto 2, que era de $60 \%$ no pré-teste, piorou enquanto durou a fase de ensino das relações para o Conjunto 1, mas aumentou abruptamente após o ensino das relações palavra ditada-figura (AB) envolvendo os estímulos do Conjunto 2.

Em síntese, apesar de alguma variabilidade entre os participantes, a tendência geral foi uma melhora nas vocalizações, tanto para os estímulos envolvidos nas tarefas de ensino, como também para os estímulos do outro conjunto, que eram mantidos em linha de base, como resultado da exposição ao ensino de discriminações condicionais. $\mathrm{O}$ efeito foi imediato em alguns casos (isto é, ocorreu após o ensino do primeiro tipo de relação), enquanto em outros ocorreu gradualmente, ao longo do ensino dos diferentes tipos de relações. $\mathrm{O}$ ensino com os estímulos do segundo conjunto geralmente manteve ou melhorou o desempenho diante dos estímulos dos dois conjuntos.

Uma decorrência do delineamento empregado é que o repertório de fala com palavras do Conjunto 1 continuou sendo avaliado (retenção) durante o ensino e as sucessivas sondas de vocalização com palavras do Conjunto 2. Observou-se que o repertório de nomear adquirido não só foi mantido, mas ainda melhorou, nos casos em que ainda havia margem para melhora.

\section{Discussão}

Todos os participantes aprenderam novas relações entre palavras ditadas e figuras (AB) e entre palavras ditadas e palavras impressas (AC) nas duas unidades de ensino, o que replica resultados de estudos anteriores com usuários de implante coclear (Almeida-Verdu et al., 2008; Bataglini et al., 2013; Golfeto, 2010).

Todos os participantes demonstraram emergência de relações não diretamente ensinadas entre figuras e palavras impressas (BC) e entre palavras impressas e figuras (CB) com pelo menos um conjunto de estímulos. A formação de classes de equivalência é o critério para a demonstra- ção de comportamento simbólico (Sidman, 1994). Esses resultados confirmam que os sons recebidos por meio do implante coclear podem compartilhar de funções simbólicas, podendo adquirir funções de controle sobre o comportamento dos implantados, assim como ocorre com pessoas com o sistema auditivo preservado (Almeida-Verdu et al., 2008). Esses dados também replicam os encontrados na literatura sobre formação de classes de equivalência com outros tipos de população, como indivíduos com déficit intelectual (Sidman, 1971) ou indivíduos com autismo e reduzido repertório verbal (Carr, Wilkinson, Blackman, \& McIlvane, 2000; ver também a revisão de Gomes, Varella, $\&$ de Souza, 2010). Embora o comportamento de leitura não tenha sido diretamente ensinado, esse comportamento tende a emergir, por derivação das contingências de ensino empregadas, que promoveram a formação de classes de equivalência entre palavra ditada, palavra escrita e figuras (de Rose, de Souza, \& Hanna, 1996; de Souza, de Rose, \& Domeniconi, 2009).

A tarefa de nomear requer desempenho sob controle de estímulos ou de relações entre eles que não estão mais presentes, exigindo memória visual, de procedimento e, sobretudo, auditiva (Catania, 1999). Todos os participantes deste estudo apresentavam maiores porcentagens de acertos em leitura do que em nomeação de figuras, como mostraram os dados do pré-teste. Todos os participantes diminuíram a discrepância entre a nomeação e a leitura após a exposição às etapas de ensino com estímulos dos dois conjuntos. Considerando-se os acertos iniciais em leitura, o progresso em nomeação pode ter derivado das extensões do controle exercido pela palavra impressa sobre a figura. Em outras palavras, no caso deste estudo, o fortalecimento das relações de equivalência entre palavra ditada, palavra escrita e figura, pode ter oferecido condições para que o repertório de falante emitido na presença da palavra impressa fosse estendido para a figura (Sidman, 2000).

Outro aspecto do delineamento pode ter favorecido o aumento na porcentagem de acertos em tarefas de nomeação. $\mathrm{O}$ delineamento de linha de base múltipla entre conjuntos de palavras, com sucessivas sondas de nomeação e de leitura, após cada etapa do ensino, pode ter permitido sucessivas oportunidades de ouvir (no treino auditivovisual) e de falar (durante as sondas), constituindo-se em um supertreino, isto é, a manutenção ou repetição do procedimento de ensino depois que o aprendiz atingiu o critério de aprendizagem. No que diz respeito à leitura e à escrita, Lee e Pegler (1982) ensinaram crianças a ler palavras apresentadas sucessivamente em cartões e testaram a escrita sob ditado; esse ciclo leitura-escrita foi repetido várias vezes e resultou em melhora gradual na composição de palavras. As autoras concluíram que o supertreino pode ter facilitado a transferência de um comportamento verbal para outro. Esta relação cíclica pode ser estendida para o ouvir e o falar, de tal forma que a melhora no desempenho de falar pode ter sido produto tanto das oportunidades de ouvir durante o ensino, como das repetidas oportunidades para o falar, intercaladas com as tarefas de ensino. Nas primeiras sondas após o ensino de algumas relações 
os participantes tenderam a emitir topografias vocais incompletas ou não correspondentes às palavras ditadas durante o procedimento; porém, o fato de terem sucessivas oportunidades de ouvir a palavra e selecioná-la (ensino), antes de ter a oportunidade de vocalizá-la, pode ter oferecido contingências de modelagem (reforço diferencial por aproximações sucessivas) da vocalização, o que levou, ao longo de sucessivos testes, a um desempenho cada vez mais semelhante ao alvo e, em alguns casos, a uma fala precisa. Se esses participantes necessitariam de mais exposições ao ciclo ouvir e falar com os mesmos estímulos verbais ou com novos conjuntos, para um fortalecimento mais generalizado de fala precisa e inteligível, são aspectos a serem explorados em novas investigações.

Outro aspecto a considerar é a possibilidade de que esses participantes estejam começando a apresentar comportamentos generalizados (ou classes de ordem superior; cf. Catania, 1999), especialmente considerando-se o uso de pseudopalavras como estímulos a serem relacionados a figuras abstratas. $\mathrm{O}$ aumento na nomeação de figuras abstratas do Conjunto 2, antes que fossem ensinadas (durante as sondas ao longo do ensino com o Conjunto 1) pode ter resultado de uma atribuição arbitrária (cf. Harrison \& Green, 1990; Saunders \& Spradlin, 1990) para a relação palavra-figura. Como o participante era sucessivamente exposto a palavras impressas e figuras, ele pode ter decodificado palavras como TIBA e ZIGO. Nas tentativas de nomeação, cada figura familiar tinha um nome definido; diante de uma figura abstrata, o participante pode ter empregado, arbitrariamente, uma das palavras lidas (com 50\% de probabilidade de acerto) e consistentemente, atribuído a outra palavra à outra figura abstrata (tato generalizado). Isto poderia explicar, pelo menos em parte, a ocorrência de um desempenho que parecia improvável e, também, a variabilidade interindividual (dada a probabilidade de acerto, enquanto um aluno, por acaso, pode ter feito a atribuição correta, outro pode ter feito a atribuição incorreta). Contudo, essas possibilidades devem ser confrontadas com evidencias empíricas, que não foram obtidas com o presente procedimento.

Em investigações futuras, o controle experimental para demonstrar os efeitos das manipulações experimentais poderia ser aumentado, buscando-se combinar o delineamento de linha de base múltipla entre participantes com o delineamento de linha de base múltipla entre conjuntos de estímulos e de múltiplas sondas.

\section{Considerações Finais}

Os resultados encontrados indicam que o fortalecimento na rede de relações entre estímulos e a classe de respostas de falar produziu melhoras na fala sob controle das figuras (nomeação). No início do estudo todos os participantes falavam as palavras experimentais sob controle do texto; embora com alguns erros, a fala era inteligível, portanto os participantes podiam emitir a resposta. Contudo, diante das figuras, a fala era bastante incompleta e distorcida; o problema parecia residir não em uma incapacidade para falar, mas na falta de controle de estímulos pela figura sobre a resposta vocal. Casos como os observados neste estudo reiteram a noção de independência funcional entre diferentes operantes verbais (Skinner, 1957), mesmo que a resposta seja a mesma, como demonstrado empiricamente em uma série de estudos (e.g., Lamarre \& Holland, 1985; Lee \& Pegler, 1982). Ao final do experimento, o controle de estímulos exercido pela palavra falada, pela palavra escrita e pela figura se tornaram equivalentes, como demonstrado pelos testes críticos $\mathrm{BC}$ e $\mathrm{CB}$; por meio desse processo, o controle de estímulos das palavras impressas sobre a fala parece ter sido transferido para as figuras, isto é, cada figura passou a controlar uma fala tão precisa quanto a palavra impressa correspondente. Por sua vez, o controle por palavra impressa e por figura parece possível devido à relação simbólica entre ambas e destas com a palavra falada, adquirido por equivalência de estímulos (de Souza et al., 2009).

O procedimento empregado demonstrou-se viável para acelerar os processos de discriminação auditiva, de estabelecimento de relações entre estímulos sonoros e outros aspectos do ambiente e de produção de fala, diminuindo desvantagens na vocalização de crianças com deficiência auditiva.

A replicação deste estudo em escala maior poderia verificar se o efeito observado é robusto o suficiente para recomendar o procedimento como uma tecnologia de ensino eficaz na reabilitação de populações com deficiência auditiva.

\section{Referências}

Almeida-Verdu, A. C., Huziwara, E. M., de Souza, D. G., de Rose, J. C., Bevilacqua, M. C., Lopes, J., Jr., ...McIlvane, W. J. (2008). Relational learning in children with deafness and cochlear implants. Journal of the Experimental Analysis of Behavior, 89, 407-427. doi:10.1901/jeab.2008-89-407

Almeida-Verdu, A. C. M., Oliveira, F. M., Battaglini, M. P., Bevilacqua, M. C., \& de Souza, D. G. (2012). Desempenho de seleção e nomeação em crianças com deficiência auditiva e implante coclear. Temas em Psicologia, 20(1), 189-202.

Almeida-Verdu, A. C. M., Souza, F. C., de Souza, D. G., \& Bevilacqua, M. C. (2009). Imitação vocal e nomeação de figuras em deficientes auditivos usuários de implante coclear: Estudo exploratório. Revista Brasileira de Análise do Comportamento, 5(1), 63-78.

Bataglini, M., Almeida-Verdu, A. C. M., \& Bevilacqua, M. C. (2013). Aprendizagem após exclusão e formação de classes em crianças com deficiência auditiva e implante coclear. Acta Comportamentalia, 21(1), 20-35.

Bevilacqua, M. C., Delgado, E. M. C., \& Moret, A. L. M. (1996). Estudos de casos clínicos de crianças do Centro Educacional do Deficiente Auditivo (CEDAU), do Hospital de Pesquisa e Reabilitação de Lesões Lábio-Palatais - USP. In O. A. Costa \& M. C. Bevilacqua (Eds.), Anais: XXI Encontro Internacional de Audiologia (p. 187). Bauru, SP: Academia Brasileira de Audiologia.

Carr, D., Wilkinson, K. M., Blackman, D., \& McIlvane, W. J. (2000). Equivalence classes in individuals with minimal verbal repertoires. Journal of the Experimental Analysis of Behavior, 74, 101-114. doi:10.1901/jeab.2000.74-101 
Anastácio-Pessan, F. L., Almeida-Verdu, A. C. M., Bevilacqua, M. C. \& de Souza, D. G. (2015). Usando o Paradigma de Equivalência para Aumentar a Correspondência na Fala de Crianças com Implante Coclear na Nomeação de Figuras e na Leitura.

Catania, A. C. (1999). Aprendizagem: Comportamento, linguagem e cognição. Porto Alegre, RS: Artes Médicas.

Da Silva, W. R., de Souza, D. G., de Rose, J. C., Lopes, J., Jr., Bevilacqua, M. C., \& McIlvane, W. J. (2006). Relational learning in deaf children with cochlear implants. Experimental Analysis of Human Behavior Bulletin, 23(24), 1-8.

De Rose, J. C., de Souza, D. G., \& Hanna, E. S. (1996). Teaching reading and spelling: Exclusion and stimulus equivalence. Journal of Applied Behavior Analysis, 29, 451-469. doi:10.1901/jaba.1996.29-451

De Souza, D. G., de Rose, J. C., \& Domeniconi, C. (2009). Applying relational operants to reading and spelling. In R. A. Refeldt \& Y. Barnes-Holmes (Eds.), Derived relational responding: Applications for learners with autism and other developmental disabilities (pp. 173-207). Oakland, CA: New Harbinger.

Dixon, L. S. (1977). The nature of control by spoken words over visual stimulus selection. Journal of the Experimental Analysis of Behavior, 27, 433-442. doi:10.1901/jeab.1977.27-433

Dube, W. V. (1991). Computer software for stimulus control research with Macintosh computers. Experimental Analysis of Human Behavior Bulletin, 9, 28-39.

Fortunato, C. A. U., Bevilacqua, M. C., \& Costa, M. P. R. (2009). Análise comparativa da linguagem oral de crianças ouvintes e surdas usuárias de implante coclear. Revista CEFAC, 11(4), 662-672. doi:10.1590/S1516-18462009000800015

Geers, A. E. (1994). Techniques for assessing auditory speech perception and lipreading enhancement in young deaf children. Volta Review, 96(5), 85-96.

Geers, A. E., Nicholas, J. G., \& Moog, J. S. (2007). Estimating the influence of cochlear implantation in language development in children. Audiological Medicine, 5, 262-273. doi:10.1080/16513860701659404

Golfeto, R. M. (2010). Compreensão e produção de fala em crianças com surdez pré-lingual usuárias de implante coclear (Tese de doutorado, Universidade Federal de São Carlos, SP, Brasil).

Gomes, C. G. S., Varella, A. A. B., \& de Souza, D. G. (2010). Equivalência de estímulos e autismo: Uma revisão de estudos empíricos. Psicologia: Teoria e Pesquisa, 26, 729-737. doi:10.1590/S0102-37722010000400017

Greer, R. D., \& Ross, D. E. (2008). Verbal behavior analysis: Inducing and expanding complex communication in children with severe language delays. Boston, MA: Allyn \& Bacon.

Hanna, E. S., de Souza, D. G., de Rose, J. C., \& Fonseca, M. (2004). Effects of delayed constructed-response identity matching on spelling of dictated words. Journal of Applied Behavior Analysis, 37(2), 223-227. doi:10.1901/jaba.2004.37223

Harrison, R. J., \& Green, G. (1990). Development of conditional and equivalence relations without differential consequences. Journal of the Experimental Analysis of Behavior, 54, 225237. doi:10.1901/jeab.1990.54-225

Houston, D. M., Stewart, J., Moberly, A., Hollich, G., \& Miyamoto, R. T. (2012). Word learning in deaf children with cochlear implants: Effects of early auditory experience. Developmental Science, 15(3), 448-461. doi:10.1111/j.14677687.2012.01140.x

Kazdin, A. E. (1982). Single-case research designs: Methods for clinical and applied settings. New York: Oxford University Press.

Kirk, K. I., Miyamoto, R. T., Ying, E. A., Perdew, A. E., \& Zuganelis, H. (2002). Cochlear implantation in young children:
Effects of age at implantation and communication mode. Volta Review, 102, 127-144.

Lamarre, J., \& Holland, J. G. (1985). The functional independence of mands and tacts. Journal of the Experimental Analysis of Behavior, 43, 5-19. doi:10.1901/jeab.1985.43-5

Lee, V. L., \& Pegler, A. (1982). Effects on spelling of training children to read. Journal of the Experimental Analysis of Behavior, 37, 311-322. doi:10.1901/jeab.1982.37-311

Lee, V. L., \& Sanderson, G. M. (1987). Some contingencies on spelling. The Analysis of Verbal Behavior, 5, 1-13.

McIlvane, W. J., Kledaras, J. B., Stoddard, L. T., \& Dube, W. V. (1990). Delayed sample presentation in MTS: Some possible advantages for teaching individuals with developmental limitations. Experimental Analysis of Human Behavior Bulletin, 8, 31-33.

McIlvane, W. J., \& Stoddard, L. T. (1981). Acquisition of matching-to-sample performances in severe mental retardation: Learning by exclusion. Journal of Mental Deficiency Research, 25, 33-48.

Melo, T. M., Moret, A. L. M., \& Bevilaccqua, M. C., (2008). Avaliação da produção de fala em crianças deficientes auditivas usuárias de Implante Coclear Multicanal. Revista da Sociedade Brasileira de Fonoaudiologia, 13(1), 45-51. doi:10.1590/S1516-80342008000100009

Mondain, M., Sillon, M., Vieu, A., Levi, A., Reuillard-Artieres, F., Deguine, O., ...Uziel, A. (2002). Cochlear implantation in prelingually deafened children with residual hearing. International Journal of Pediatric Otorhynolaryngology, 63(2), 91-97. doi:10.1016/S0165-5876(01)00638-3

Passarelli, A. C. P. M., Oliveira, T. P., Golfeto, R. M., Cardinali, R., Rezende, J. V., \& Fenner, M. C. (2013). Discriminação auditivo-visual com pistas orofaciais em crianças deficientes auditivas. Acta Comportamentalia, 21(2), 175-192.

Saunders, K. J., \& Spradlin, J. E. (1990). Conditional discrimination in mentally retarded adults: The development of generalized skills. Journal of the Experimental Analysis of Behavior, 54, 225-237. doi:10.1901/jeab.1989.52-1

Sidman, M. (1971). Reading and auditory-visual equivalences. Journal of Speech and Hearing Research, 14, 5-13. doi:10.1044/jshr.1401.05

Sidman, M. (1994). Equivalence relations and behavior: A research story. Boston, MA: Authors Cooperative.

Sidman, M. (2000). Equivalence relations and the reinforcement contingency. Journal of the Experimental Analysis of Behavior, 74, 127-146. doi:10.1901/jeab.2000.74-127

Sidman, M., \& Tailby, W. (1982). Conditional discriminations vs. matching to sample: An expansion of the testing paradigm. Journal of the Experimental Analysis of Behavior, 37, 5-22.

Skinner, B. F. (1957). Verbal behavior. New York: AppletonCentury-Crofts.

Souza, F. C., Almeida-Verdu, A. C. M., \& Bevilacqua, M. C. (2013). Ecóico e nomeação de figuras em crianças com deficiência auditiva pré-lingual com implante coclear. Acta Comportamentalia, 21(3), 325-339.

Svirsky, M. A., Robbins, A. M., Kirk, K. I., Pisoni, D. B., \& Miyamoto, R. T. (2000). Language development in profoundly deaf children with cochlear implants. Psychological Science, 11(2), 153-158. doi:10.1111/1467-9280.00231 